\title{
Modelling directionality for paleoclimatic time series
}

\author{
M. M. Mansor ${ }^{1} \quad$ F. L. Mohd. Isa ${ }^{2} \quad$ D. A. Green ${ }^{3}$ \\ A. V. Metcalfe ${ }^{4}$
}

(Received 2 January 2016; revised 26 May 2016)

\begin{abstract}
The ice core time series from Vostok Station in Antarctica and the North Greenland Ice Core Project have seasonal variation corresponding to the Milankovitch cycles. After removing these cycles, and interpolating to equal time intervals, stationary time series models are fitted. The series show clear directionality and this feature is modelled by either non-Gaussian errors or non-linear time series models. Threshold autoregressive models are fitted by penalized least squares and compared with non-threshold autoregressive models. Since both ice core time series are reasonably modelled as first order autoregressive series with parameters close to one, directionality will arise from non-symmetric error distributions. However, two regime threshold
\end{abstract}

http://journal.austms.org.au/ojs/index.php/ANZIAMJ/article/view/10415 gives this article, (C) Austral. Mathematical Soc. 2016. Published June 17, 2016, as part of the Proceedings of the 12th Biennial Engineering Mathematics and Applications Conference. ISSN 1446-8735. (Print two pages per sheet of paper.) Copies of this article must not be made otherwise available on the internet; instead link directly to this URL for this article. 
autoregressive models, of order one and two for Greenland and Vostok, respectively, give an improved match to the observed directionality and a reduced sum of squared residuals. Realizations from the threshold autoregressive models are noticeably different from the non-threshold models. Since the non-threshold models are a restricted case of the threshold models, and the threshold models are a better fit to the observed time series, threshold models should provide more realistic realizations.

\section{Contents}

1 Introduction

2 Detecting directionality

C68

3 Modelling directionality

C72

3.1 Modelling directionality for NGRIP series . . . . . . . C73

3.2 Modelling directionality for Vostok series . . . . . . . . C74

3.3 TAR model with penalized least squares . . . . . . . C75

4 Climate change simulation

C77

5 Conclusion

C78

References

C79

\section{Introduction}

Ice cores contain information about the history of Earth's climate. This information is derived from the ancient impurities trapped in the ice for thousands of years, including air bubbles, volcanic ash and soot. The proportion of dissolved oxygen isotope-18 to dissolved oxygen isotope-16 in ice, 
and the proportion of deuterium (heavy water) in ice, are closely related to temperature. The local temperature is deduced from the proportion of oxygen-18 or from the relative amount of deuterium in the water molecules of the ice compared with seawater.

In this article we investigate paleoclimatic time series from the North Greenland Ice Core Project (NGRIP) which begins 122900 years before present (BP) [7], and the Vostok ice core record which begins 422766 years BP [8]. The NGRIP data includes $\delta^{18} \mathrm{O}$ which is the ratio of oxygen isotope-18 $\left({ }^{18} \mathrm{O}\right)$ to oxygen isotope-16 $\left({ }^{16} \mathrm{O}\right)$ at 50 year intervals. The Vostok time series data is the deuterium (an isotope of hydrogen) content $\delta \mathrm{D}$ as a percentage of Standard Mean Ocean Sea Water (SMOW) at approximately 50 year intervals.

Both time series contain seasonal variations that corresponds to the Milankovitch cycles, long term variations in the Earth's orbit that have been affecting the Earth's climate change for aeons. We remove the effect of Milankovitch cycles in both series by fitting multiple regression to obtain the deseasonalized time series. The deseasonalized NGRIP (ds NGRIP series) is equally spaced at 50 year intervals. However, the deseasonalized Vostok (ds Vostok series) is unequally spaced due to missing values, particularly in the early record. As further analyses are based on evenly spaced time series, we applied linear interpolation at 50 year increments to obtain the deseasonalized and interpolated Vostok (dsi Vostok series).

\section{Detecting directionality}

A stationary time series model $\left\{X_{t}\right\}$ for time $t=1,2, \ldots, n$ is reversible if the joint distribution of $X_{t}, X_{t+1}, \ldots, X_{t+r}$ is equal to the joint distribution of $X_{t+r}, X_{t+r-1}, \ldots, X_{t}$ for all $r=1,2, \ldots$ [2]. A stationary time series model is directional if these joint distributions differ. If a stationary time series model is reversible, then it will not be possible to distinguish realisations plotted against time order from those realisations plotted in reverse time order, that 
is, against time-to-go ${ }^{1}$. If a stationary time series model is directional, then, in principle, there will be qualitative differences between realisations plotted in time order and the same realisations plotted against time-to-go. However, the difference may not be discernible from a single time series, so a formal statistical test is required to detect and quantify the directionality.

Stationary linear time series models with Gaussian white noise are reversible, whereas non-linear time series models and linear models driven by nonGaussian white noise are directional [3]. Directionality is clearly implicit in a trend and in asymmetric seasonal patterns, so directionality is only of interest in itself when considered in its own right for stationary time series models. If a time series appears to have a trend or seasonal effects, then these should be identified and removed before considering directionality.

Directionality is visible in many stationary time series from various disciplines including environmental science. We compare a plot against time with a plot against time-to-go for the ds NGRIP series (Figure 1) and for the dsi Vostok series (Figure 2). The ds NGRIP appears to be a realisation of a stationary time series model, and there is evidence against a null hypothesis of a unit root (Dickey-Fuller test, probability $\mathrm{P}<0.01$ ) to support this claim. The ds NGRIP series has a tendency for sharp increases before the peaks followed by gradual decreases to the troughs when plotted against time, and gradual increases before the peaks followed by sharp decreases to the troughs when plotted against time-to-go.

The dsi Vostok series also appears to be a realisation of a stationary time series model, and the Dickey-Fuller test again provides evidence against a hypothesis of a unit root $(\mathrm{P}<0.01)$. There is a slight tendency for rapid increases to be followed by slower decreases, but this is more apparent in the first half of the series which is considerably smoother because many values are interpolated. To avoid the possibility that our findings are heavily influenced by interpolation, we focus on the second half of the dsi Vostok series (dsi $2 \mathrm{H}$ Vostok series) for further analyses.

${ }^{1}$ If time $t$ runs from 1 to $n$, then the time-to-go is $n-t$, and runs from $n-1$ to 0 . 
Figure 1: The ds NGRIP series (top) against time with scale in years BP; and (bottom) against time-to-go in 50 year units.

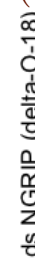

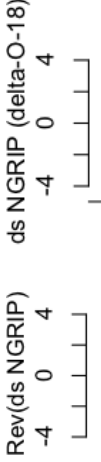

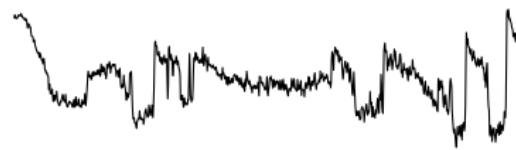

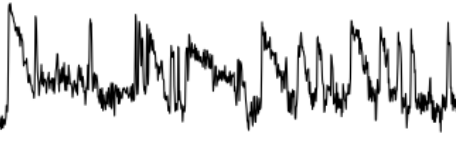

120000

80000

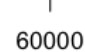

40000

Time (50-year BP)
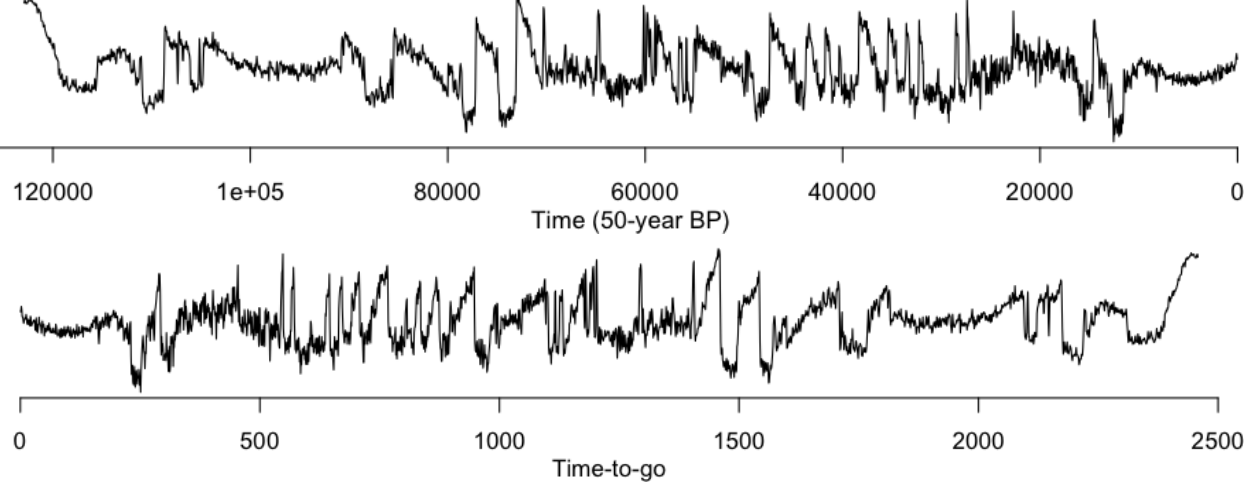

Figure 2: The dsi Vostok series (top) against time with scale in years BP; and (bottom) against time-to-go in 50 year units.

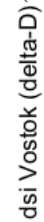
क
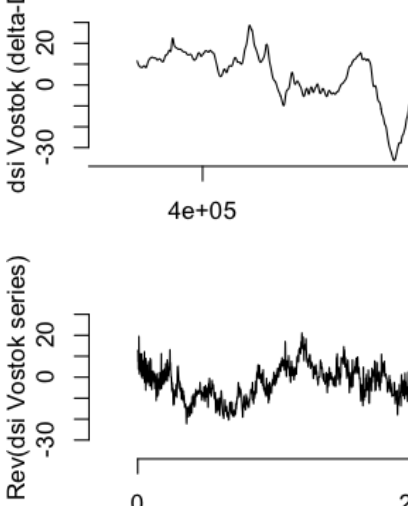

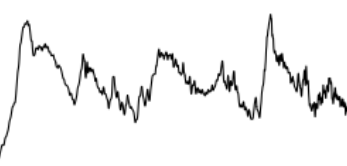

$3 e+05$

$2 \mathrm{e}+05$
Time $(50$-year BP)

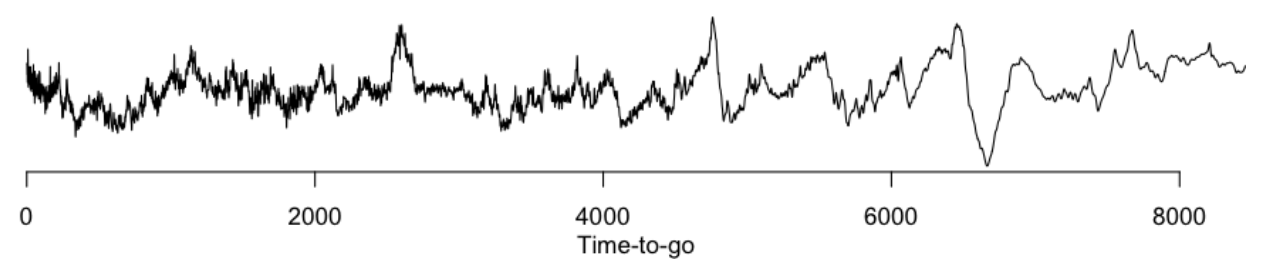


Table 1: Statistics for ds NGRIP and dsi 2H Vostok series.

\begin{tabular}{lrr} 
Statistic & $\operatorname{NGRIP}\left(\delta^{18} \mathrm{O}\right)$ & $\operatorname{Vostok}(\delta \mathrm{D})$ \\
\hline Time interval (years) & 50 & 50 \\
No. obs., $\mathrm{n}$ & 2459 & 4229 \\
Mean, $\bar{x}$ & 0.00 & -2.00 \\
Standard dev., $s$ & 1.51 & 7.96 \\
Directionality, $\hat{\gamma}_{\text {dif }}$ & 0.61 & 0.18
\end{tabular}

The next step is to quantify the apparent directionality in the data and determine whether or not it can plausibly be attributed to chance. A general indicator of directionality is the product moment skewness of first differences [3] estimated by

$$
\hat{\gamma}_{\text {dif }}=\frac{\sum_{t=1}^{n}\left(y_{t}-\bar{y}\right)^{3} /(n-1)}{\left[\sum_{t=1}^{n}\left(y_{t}-\bar{y}\right)^{2} /(n-1)\right]^{3 / 2}},
$$

where $\left\{y_{t}\right\}=\left\{x_{t}\right\}-\left\{x_{t-1}\right\}$ for $t=2,3, \ldots, n, \bar{y}$ is the mean of $\left\{y_{t}\right\}$, and $\left\{x_{t}\right\}$ is the observed time series. For example, a distribution of first differences $y_{t}$ of the NGRIP series (that has rapid increases followed by more gradual recessions) tends to have more small negative differences than positive differences. Although the positive differences are fewer, they include outlying positive differences corresponding to the rapid increases. It follows that $y_{t}$ is positively skewed with a longer tail on the right side of the distribution (negative median and mean value to the right of the median). Non-zero skewness indicates directionality or asymmetry in time of a time series. We refer to $\hat{\gamma}_{\text {dif }}$ as directionality. Directionalities in the ds NGRIP and dsi $2 \mathrm{H}$ Vostok series are shown in Table 1, together with the lengths, means and standard deviations of these series. The positive directionalities correspond to the relatively rapid increases and slow recessions seen in Figures 1 and 2.

Next, we determine the significance level of the indicator of directionality using a randomization test based on autoregressive models $A R(p)$, where the $p$ order is determined by the minimum Akaike information criterion (AIC), with 
Gaussian white noise for the errors. The probability of a directionality with absolute magnitude greater than 0.61 in Gaussian white noise in realisations of length 2459 (the number of 50 year intervals in the ds NGRIP data) is less than 0.001, as determined from AR(5) where five is selected by AIC. So there is strong evidence of directionality in the ds NGRIP series $(P<0.001)$. Similarly, there is strong evidence of directionality in the dsi $2 \mathrm{H}$ Vostok series $(\mathrm{P}<0.001$, based on $\mathrm{AR}(36))$.

Directionality in time series has several implications. Directionality provides evidence of complex feedbacks after shocks or occasional extreme events $[9,4$, e.g.]. In the context of paleoclimatic series, extreme events include earth quakes, volcanoes, and meteorite strikes. Moreover, directionality may indicate that non-linear time series models are appropriate and these should provide more accurate forecasts and more realistic ensembles of scenarios [3, e.g.].

\section{$3 \quad$ Modelling directionality}

Reproduction of directionality in time series is done by introducing nonGaussian errors or by using a non-linear model [2,3] with either Gaussian or non-Gaussian error distributions. Non-Gaussian errors include: asymmetric probability distributions, such as Gumbel, three-parameter Weibull, beta and back-to-back Weibull; and symmetric probability distributions such as Student's-t. However, the Student's-t distribution needs to have high kurtosis (low degree of freedom) to inculcate noticeable directionality in the linear model [4].

We fit the observed ds NGRIP and dsi $2 \mathrm{H}$ Vostok series to AR models,

$$
\left(X_{t}-\mu\right)=\sum_{i=1}^{p} \alpha_{i}\left(X_{t-i}-\mu\right)+\epsilon_{t},
$$

where the mean $\mu$ and the coefficients $\alpha_{1}, \ldots, \alpha_{p}$ are the parameters to be estimated, and $\epsilon_{\mathrm{t}}$ is a sequence of independent zero mean random errors. To 
Table 2: Estimated standard deviation of residuals $\hat{\sigma}_{\text {err }}$ for AR models.

\begin{tabular}{l|rr|rr} 
Order & \multicolumn{2}{|c}{ NGRIP $\left(\delta^{18} \mathrm{O}\right)$} & \multicolumn{2}{c}{ Vostok $(\delta \mathrm{D})$} \\
\hline 0 & & $s=1.51$ & & $s=7.96$ \\
1 & $\operatorname{AR}(1)$ & $\hat{\sigma}_{\text {err }}=0.6203$ & $\operatorname{AR}(1)$ & $\hat{\mathrm{o}}_{\text {err }}=1.6134$ \\
2 & $\operatorname{AR}(2)$ & $\hat{\mathrm{o}}_{\text {err }}=0.6200$ & $\operatorname{AR}(2)$ & $\hat{\mathrm{o}}_{\text {err }}=1.4907$ \\
AIC & $\operatorname{AR}(5)$ & $\hat{\mathrm{o}}_{\text {err }}=0.6173$ & $\operatorname{AR}(36)$ & $\hat{\mathrm{o}}_{\text {err }}=1.3398$
\end{tabular}

fit the models we use ordinary least squares, and we assess the goodness of fit by comparing the estimated standard deviation of the residuals $\hat{\sigma}_{\text {err }}$ with the marginal standard deviation $s$ of the observed time series.

Table 2 presents the fitting results and suggests that the simple AR(1) model is a reasonable first approximation for the ds NGRIP series, and similarly the AR(2) model for the dsi $2 \mathrm{H}$ Vostok series. In both cases, the standard deviation of the residuals $\hat{\sigma}_{\text {err }}$ are considerably lower than the marginal standard deviations of the ice core time series $s$, and the further decreases in the residuals for AIC model are negligible.

\subsection{Modelling directionality for NGRIP series}

We model directionality for the ds NGRIP series using the AR(1) model and threshold autoregressive model (TAR) of order one, TAR(1), fitted by (nonlinear) least squares, with Gaussian errors (GE) and resampled residuals (RE), randomly with replacement,. The TAR(1) model is

$$
\left(X_{t}-\mu\right)= \begin{cases}\alpha_{\mathrm{u}}\left(X_{t-1}-\mu\right)+\epsilon_{t} & \text { if }\left(X_{t-1}-\mu\right)>T, \\ \alpha_{L}\left(X_{t-1}-\mu\right)+\epsilon_{t} & \text { if }\left(X_{t-1}-\mu\right)<T,\end{cases}
$$

where the mean $\mu$ and the coefficients $\alpha_{\mathrm{U}}$ and $\alpha_{\mathrm{L}}$ are parameters to be estimated, $\epsilon_{t}$ is a sequence of independent zero mean random errors, and $\mathrm{T}$ is the threshold which is calculated here as the upper 0.80 quantile of the marginal distribution of the ds NGRIP time series. 
Table 3: Comparison of directionality $\hat{\gamma}_{\text {sim }}$, mean $\bar{\chi}_{\text {sim }}$ and standard deviation $\bar{x}_{\text {sim }}$ from realisations (length $\left.10^{5}\right)$ of $\operatorname{AR}(1)$ and $\operatorname{TAR}(1)$ fitted to the ds NGRIP series with target values $\hat{\gamma}=0.61, \bar{x}=0.00$ and $s=1.51$.

\begin{tabular}{l|rrrr} 
& $\operatorname{AR}(1)+\mathrm{GE}$ & $\mathrm{TAR}(1)+\mathrm{GE}$ & $\mathrm{AR}(1)+\mathrm{RE}$ & $\mathrm{TAR}(1)+\mathrm{RE}$ \\
\hline$\hat{\gamma}_{\mathrm{sim}}$ & -0.004 & -0.002 & 0.630 & 0.632 \\
$\bar{\chi}_{\text {sim }}$ & -0.016 & 0.055 & -0.005 & -0.008 \\
$\mathrm{~s}_{\mathrm{sim}}$ & 1.496 & 1.479 & 1.508 & 1.507
\end{tabular}

The directionality arises from the distribution of the errors rather than the non-linearity of the TAR(1) model (Table 3), which has almost identical parameters above and below the 0.80 quantile threshold (Table 6, second row).

\subsection{Modelling directionality for Vostok series}

In the case of the dsi $2 \mathrm{H}$ Vostok series, we model directionality using $\operatorname{AR}(2)$ and TAR(2) models. The TAR(2) model is

$$
\left(X_{t}-\mu\right)= \begin{cases}\alpha_{1 u}\left(X_{t-1}-\mu\right)+\alpha_{2 u}\left(X_{t-2}-\mu\right)+\epsilon_{t} & \text { if }\left(X_{t-1}-\mu\right)>T, \\ \alpha_{1 L}\left(X_{t-1}-\mu\right)+\alpha_{2 L}\left(X_{t-2}-\mu\right)+\epsilon_{t} & \text { if }\left(X_{t-1}-\mu\right)<T,\end{cases}
$$

where the mean $\mu$ and the coefficients $\alpha_{1 \mathrm{u}}, \alpha_{2 \mathrm{U}}, \alpha_{1 \mathrm{~L}}$ and $\alpha_{2 \mathrm{~L}}$ are parameters to be estimated, $\epsilon_{t}$ is a sequence of independent zero mean random errors, and $\mathrm{T}$ is the threshold which is calculated as the upper 0.80 quantile of the marginal distribution of the dsi $2 \mathrm{H}$ Vostok series.

Results in Table 4 show both the errors and non-linearity of the TAR(2) induce directionality, but the TAR(2) with RE has more directionality then the original series. 
Table 4: Comparison of directionality $\hat{\gamma}_{\text {sim }}$, mean $\bar{\chi}_{\text {sim }}$ and standard deviation $s_{\text {sim }}$ of simulation of length $10^{5}$ from $\operatorname{AR}(2)$ and $\operatorname{TAR}(2)$ fitted to the dsi $2 \mathrm{H}$ Vostok series with target values $\hat{\gamma}=0.18, \bar{x}=-2.00$ and $s=7.96$.

\begin{tabular}{c|rrrr} 
& $\operatorname{AR}(2)+\mathrm{GE}$ & $\mathrm{TAR}(2)+\mathrm{GE}$ & $\mathrm{AR}(2)+\mathrm{RE}$ & $\mathrm{TAR}(2)+\mathrm{RE}$ \\
\hline $\bar{\gamma}_{\mathrm{sim}}$ & -0.008 & 0.021 & 0.123 & 0.318 \\
$\bar{\chi}_{\text {sim }}$ & -2.117 & -2.725 & -1.754 & -1.630 \\
$\mathrm{~s}_{\mathrm{sim}}$ & 7.875 & 8.141 & 7.944 & 8.295
\end{tabular}

\subsection{TAR model with penalized least squares}

In order to improve the agreement between the simulated directionality and the directionality observed in the original time series (target directionality), we estimate parameters of the TAR(1) model given in equation (3) for the ds NGRIP series and parameters of the TAR(2) model given in equation (4) for the dsi $2 \mathrm{H}$ Vostok series using penalized least squares (PLS). The objective is

$$
\omega=\sum_{t=p+1}^{n} r_{t}^{2}+\phi\left(\hat{\gamma}_{\text {observed }}-\hat{\gamma}_{\text {simulated }}\right)^{2},
$$

where $\left\{r_{t}\right\}$ are residuals from the fitted models and $\phi$ is the weight given to minimise discrepancy between the target directionality and the simulated directionality. Constraints on the stability for TAR(1)[PLS] are $-1<\alpha_{\mathrm{L}}$ and $\alpha_{\mathrm{U}}<1$; and for TAR(2)[PLS] are $\alpha_{2}>-1, \alpha_{1}+\alpha_{2}<1$ and $\alpha_{1}-\alpha_{2}>-1$ [1]. Errors for the TAR[PLS] models are resampled residuals from the AR model (RE1) and resampled residuals from the TAR model (RE2).

The TAR(1) is fitted using PLS with errors randomly sampled with replacement from the residuals of a previously fitted AR(1) model. For the ds NGRIP series, this TAR(1)[PLS] gives a very close agreement to the target directionality, but at the expense of a marginal standard deviation that is less than that observed (Table 5).

Table 6 shows that the AR(1) model (with resampled residuals that preserves the marginal standard deviation and is close to the target directionality) has 
Table 5: Results from realisations (length $10^{5}$ ) of TAR[PLS] models compared to target values.

\begin{tabular}{l|r|rr} 
ds NGRIP & & TAR(1)[PLS, RE1] & TAR(1)[PLS, RE2] \\
\hline$\hat{\gamma}=0.61$ & $\hat{\gamma}_{\text {sim }}$ & 0.609 & 0.579 \\
$\bar{\chi}=0.00$ & $\bar{\chi}_{\text {sim }}$ & -0.013 & -0.008 \\
$\mathrm{~s}=1.51$ & $\mathrm{~s}_{\text {sim }}$ & 1.317 & 1.339 \\
& $\phi$ & $10^{6}$ & $10^{6}$ \\
\hline dsi 2H Vostok & & TAR(2)[PLS, RE1] & TAR(2)[PLS, RE2] \\
\hline$\hat{\gamma}=0.18$ & $\hat{\gamma}_{\text {sim }}$ & 0.177 & 0.115 \\
$\bar{\chi}=-2.00$ & $\bar{\chi}_{\text {sim }}$ & -2.113 & -2.084 \\
$\mathrm{~s}=7.96$ & $s_{\text {sim }}$ & 7.669 & 7.793 \\
& $\phi$ & $10^{7}$ & $10^{7}$
\end{tabular}

Table 6: Fitting detailed: estimated coefficients and $\hat{\sigma}_{\text {err }}$ for each model.

\begin{tabular}{l|r|r} 
Model & Estimated parameters for ds NGRIP & $\hat{\sigma}_{\text {err }}$ \\
\hline $\operatorname{AR}(1)$ & $\hat{\alpha}=0.91$ & 0.620 \\
$\operatorname{TAR}(1)$ & $\hat{\alpha}_{\mathrm{U}}=0.92, \hat{\alpha}_{\mathrm{L}}=0.90$ & 0.614 \\
$\operatorname{TAR}(1)[\mathrm{PLS}, \mathrm{RE} 1]$ & $\hat{\alpha}_{\mathrm{U}}=0.86, \hat{\alpha}_{\mathrm{L}}=0.89$ & 0.617 \\
$\operatorname{TAR}(1)[\mathrm{PLS}, \mathrm{RE} 2]$ & $\hat{\alpha}_{\mathrm{U}}=0.88, \hat{\alpha}_{\mathrm{L}}=0.90$ & 0.615 \\
\hline Model & Estimated parameters for dsi $2 \mathrm{H}$ Vostok & $\hat{\mathrm{\sigma}}_{\text {err }}$ \\
\hline $\operatorname{AR}(2)$ & $\hat{\alpha}_{1}=1.4, \hat{\alpha}_{2}=-0.38$ & 1.491 \\
$\operatorname{TAR}(2)$ & $\hat{\alpha}_{1 \mathrm{U}}=1.2, \hat{\alpha}_{2 \mathrm{U}}=-0.18, \hat{\alpha}_{1 \mathrm{~L}}=1.5, \hat{\alpha}_{2 \mathrm{~L}}=-0.50$ & 1.444 \\
$\operatorname{TAR}(2)[\mathrm{PLS}, \mathrm{RE} 1]$ & $\hat{\alpha}_{1 \mathrm{U}}=1.6, \hat{\alpha}_{2 \mathrm{U}}=-0.71, \hat{\alpha}_{1 \mathrm{~L}}=1.9, \hat{\alpha}_{2 \mathrm{~L}}=-0.91$ & 1.641 \\
$\operatorname{TAR}(2)[\mathrm{PLS}, \mathrm{RE} 2]$ & $\hat{\alpha}_{1 \mathrm{U}}=1.3, \hat{\mathrm{Q}}_{2 \mathrm{U}}=-0.35, \hat{\alpha}_{1 \mathrm{~L}}=1.4, \hat{\alpha}_{2 \mathrm{~L}}=-0.37$ & 1.467
\end{tabular}


errors with standard deviation 0.620 that is only slightly higher than that of the TAR(1)[PLS,RE2] (0.615). The time series is long and a Monte Carlo simulation test indicates that TAR(1)[PLS,RE2] is a statistical improvement in terms of the standard deviation of errors $(\mathrm{P}<0.001)$. However, the simulated marginal standard deviation of 1.34 is substantially lower than the marginal standard deviation of 1.51 in the observed time series. These results highlight the limitations of relying on any single measure of goodness of fit when comparing linear and non-linear models. For NGRIP the AR(1) model, or almost equivalently the TAR(1) model, fitted without penalty, seem the best models of those considered.

For the dsi 2H Vostok series the TAR(2) model fitted by PLS offers a potential improvement on the AR(2) model, in some respects at least. The choice of error distribution affects the fit through the penalty term, because the errors determine the simulated directionality. If resampled errors after fitting the AR(2) model are used, then the directionality is matched (to two decimal places) and the marginal standard deviation of 7.67 is reasonably close to the observed 7.96. For the errors, the estimated standard deviation of 1.641 is rather higher than that of the $\operatorname{AR}(2)$ model which is 1.491. If resampled errors after fitting the TAR(2) model are used, then the estimated standard deviation of errors is reduced to 1.467 but the directionality is matched slightly better by the AR(2) model. There is no clear best model amongst the three.

\section{Climate change simulation}

Although the choice of best fitting model may be equivocal, there is a difference in terms of simulating up to ten steps ahead. This is demonstrated in Table 7 for 1000 simulations up to ten steps ahead using the AR(1) and TAR(1)[PLS,RE2] models.

For the simulation: a back-to-back Weibull distribution was fitted to the residuals of the AR(1) model; back-to-back Weibull distributions were fitted 
Table 7: Upper 1\%, median and lower $1 \%$ of $n$-step ahead predictions using $\operatorname{AR}(1)$ and TAR(1)[PLS,RE2] models for the ds NGRIP series.

\begin{tabular}{r|rr|rr|rr} 
& \multicolumn{2}{|c|}{ Upper $1 \%$} & \multicolumn{2}{|c}{ Median } & \multicolumn{2}{|c}{ Lower $1 \%$} \\
Step & AR & TAR & AR & TAR & AR & TAR \\
\hline 1 & 2.68 & 3.31 & -0.03 & -0.12 & -2.83 & -3.09 \\
2 & 3.05 & 3.41 & -0.10 & -0.14 & -2.78 & -3.03 \\
3 & 4.03 & 4.01 & -0.10 & -0.11 & -3.37 & -3.83 \\
4 & 5.12 & 4.55 & -0.10 & -0.07 & -4.83 & -4.73 \\
5 & 6.09 & 5.13 & -0.10 & -0.03 & -5.92 & -5.73 \\
6 & 6.97 & 5.60 & -0.13 & 0.01 & -6.90 & -6.73 \\
7 & 7.47 & 6.09 & -0.12 & 0.01 & -8.03 & -7.38 \\
8 & 8.09 & 6.73 & -0.11 & 0.03 & -9.13 & -8.13 \\
9 & 8.85 & 7.05 & -0.10 & 0.05 & -10.2 & -8.79 \\
10 & 9.60 & 7.45 & -0.11 & 0.06 & -10.9 & -9.39
\end{tabular}

to the TAR(1) model residuals when $x_{t}$ was below the threshold, and to the TAR( 1$)$ residuals when $x_{t}$ was above the threshold. The TAR(1) model prediction intervals were wider than the AR(1) up to three steps ahead but narrower for further steps.

\section{Conclusion}

There is clear directionality in the ds NGRIP series and statistically significant directionality in the dsi $2 \mathrm{H}$ Vostok series. The ds NGRIP series is approximated as a realisation of an $\operatorname{AR}(1)$ time series model with $\alpha=0.91$, which has first differences which are close to the errors. It follows that the directionality as measured by skewness of the differences is largely determined by the skewness of the errors. The dsi $2 \mathrm{H}$ Vostok series is roughly approximated as a realisation of an AR(1) time series model with $\alpha=0.98$ and it again follows that the directionality mainly follows the skewness of the errors. The 
ds NGRIP series at 50 year time steps is quite well modelled as a realisation of an AR(1) process with non-Gaussian errors. The non-Gaussian errors allow for some catastrophic events and the model, at least, seems stable.

Such a simple model is not satisfactory for the Vostok series. The residuals after fitting an AR(2) model still show some degree of autocorrelation. An AR(36) model is needed to obtain residuals that appear uncorrelated. The partial improvements offered by TAR models suggest that there may be more substantial non-linear effects in the Vostok series. A high order $\operatorname{AR}(p)$ model with thresholds for coefficients up to some smaller lag might provide a substantial improvement on the models considered here. However, such a complex empirical model may not provide much insight into the underlying physical processes. We also found that TAR models with penalized least squares are reproducible models for modelling directionality in stationary time series [3, 5, 6, e.g.].

Acknowledgements Maha Mansor thanks the Majlis Amanah Rakyat for an education sponsorship at the University of Adelaide, and the CSIRO Student Support Scheme for sponsoring the presentation of this work at the 12th Engineering Mathematics and Applications Conference (EMAC 2015) in Adelaide. We also thank NGRIP members [7] and Petit et al. [8] for data.

\section{References}

[1] C. Chatfield. The Analysis of Time Series: An Introduction. CRC Press, 2004. https://www.crcpress.com/

The-Analysis-of-Time-Series-An-Introduction-Sixth-Edition/ Chatfield/p/book/9781584883173 C75

[2] A. J. Lawrance. Directionality and reversibility in time series. Int. Stat. Rev. 59(1):67-79, 1991. doi:10.2307/1403575 C68, C72 
[3] M. M. Mansor, M. E. Glonek, D. A. Green and A. V. Metcalfe. Threshold autoregressive models for directional time series. In I. Rojas and H. Pomares (Eds.), Time Series Analysis and Forecasting Selected Contributions from the ITISE Conference (ITISE 2015). pp. 13-25, 2016. doi:10.1007/978-3-319-28725-6 C69, C71, C72, C79

[4] M. M. Mansor, M. E. Glonek, D. A. Green and A. V. Metcalfe. Modelling directionality in stationary geophysical time series. International work-conference on Time Series (ITISE 2015). http://www.researchgate.net/publication/281835075 C72

[5] M. M. Mansor, D. A. Green and A. V. Metcalfe. Modelling and simulation of directional financial time series. Proceedings of the 21st International Congress on Modelling and Simulation (MODSIM 2015), pp. 1022-1028, 2015.

http://www.mssanz.org.au/modsim2015/E4/mansor.pdf C79

[6] M. M. Mansor, D. A. Green and A. V. Metcalfe. Directionality and volatility in electroencephalogram time series. Proceedings of the 2nd International Conference on Mathematical Sciences and Statistics (ICMSS 2016), AIP Conf. Proc. 1739:020080, 2016. doi:10.1063/1.4952560 C79

[7] North Greenland Ice Core Project members. High-resolution record of Northern Hemisphere climate extending into the last interglacial period. Nature, 431:147-151, 2004. doi:10.1038/nature02805 C68, C79

[8] J. R. Petit, J. Jouzel, D. Raynaud, N. I. Barkov, J.-M. Barnola, I. Basile, M. Bender, J. Chappellaz, M. Davis, G. Delaygue, M. Delmotte, V. M. Kotlyakov, M. Legrand, V. Y. Lipenkov, C. Lorius, L. Pepin, C. Ritz, E. Saltzman and M. Stievenard. Climate and atmospheric history of the past 420,000 years from the Vostok ice core, Antarctica. Nature, 399:429-436, 1999. doi:10.1038/20859 C68, C79

[9] S. Soubeyrand, C. E. Morris and E. K. Bigg. Analysis of fragmented time directionality in time series to elucidate feedbacks in climate data. 
Environ. Modell. Softw. 61:78-86, 2014. doi:10.1016/j.envsoft.2014.07.003 $\mathrm{C} 72$

\section{Author addresses}

1. M. M. Mansor, School of Mathematical Sciences, University of Adelaide, South Australia 5005, Australia.

mailto:mohdmahayaudin.mansor@adelaide.edu.au

2. F. L. Mohd. Isa, School of Mathematical Sciences, University of Adelaide, South Australia 5005, Australia.

3. D. A. Green, School of Mathematical Sciences, University of Adelaide, South Australia 5005, Australia.

4. A. V. Metcalfe, School of Mathematical Sciences, University of Adelaide, South Australia 5005, Australia. 